The growth of bismuth and antimony overlayers on $\ln P(110)$

C. Stephens, D. R. T. Zahn, K. Fives, R. Cimino, W. Braun, and I. T. McGovern

Citation: Journal of Vacuum Science \& Technology B: Microelectronics Processing and Phenomena 8, 674 (1990); doi: 10.1116/1.584994

View online: https://doi.org/10.1116/1.584994

View Table of Contents: https://avs.scitation.org/toc/jvm/8/4

Published by the American Institute of Physics 


\title{
The growth of bismuth and antimony overlayers on $\ln P(110)$
}

\author{
C. Stephens \\ Department of Pure and Applied Physics, Trinity College, Dublin 2, Ireland
}

D.R.T. Zahn

Institute für Festkörperphysik, Technische Universität, Hardenbergstaße 36, D-1000, Berlin 12, Germany

K. Fives

Department of Pure and Applied Physics, Trinity College, Dublin 2, Ireland

R. Ciminoa)

Fritz-Haber-Institute der M.P.G., Faradayweg 4-6, D-1000, Berlin 33, Germany

W. Braun

Berliner Speicherring Gesellschaft für Synchrotronstrahlung m.b.H (BESSY), Lentzeallee 100, D-1000,

Berlin 33, Germany

\section{T. McGovern}

Department of Pure and Applied Physics, Trinity Colletge, Dublin 2, Ireland

(Received 30 January 1990; accepted 12 April 1990)

The adsorption of $\mathrm{Bi}$ and $\mathrm{Sb}$ on clean cleaved $\operatorname{InP}(110)$ surfaces has been studied with soft $\mathrm{X}$-ray photoemission spectroscopy at the BESSY synchrotron radiation source in Berlin. Bi and Sb were deposited onto ultrahigh vacuum cleaved $\operatorname{InP}(110)$ surfaces at substrate temperatures of $300 \mathrm{~K}$ [room temperature (RT)] and $120 \mathrm{~K}$ [low temperature (LT)]. In this paper we focus on the evolution of the overlayer emission intensities as a function of coverage. Spectra of the $\mathrm{Bi} 5 d$ and $\mathrm{Sb} 4 d$ core level emission lines were taken from the submonolayer regime up to coverages of approximately 60 and 20 monolayers (ML) of $\mathrm{Bi}$ and $\mathrm{Sb}$, respectively. The spectra were then deconvoluted using a core level fitting routine. In all cases, good agreement between the experimental and fitted curves was achieved by introducing two chemically shifted components of the $\mathrm{Bi} 5 d$ and $\mathrm{Sb} 4 d$ core level emission which are interpreted in terms of $\mathrm{Bi}(\mathrm{Sb})$-substrate and $\mathrm{Bi}(\mathrm{Sb})-\mathrm{Bi}(\mathrm{Sb})$ bonds. However, for $\mathrm{RT}$ deposition, the evolution of the $\mathrm{Bi} 5 d$ and $\mathrm{Sb} 4 d$ spectra is significantly different in the coverage range below $2 \mathrm{ML}$. In the $\mathrm{Bi}$ case the adatom-substrate component grows first, and only after about $0.5 \mathrm{ML}$ is the $\mathrm{Bi}-\mathrm{Bi}$ component observed. The two components have equal intensity at about $2 \mathrm{ML}$. In contrast, the two components in the $\mathrm{Sb} 4 d$ spectra grow with equal intensity until $1 \mathrm{ML}$. The spectra for higher coverages for both systems are dominated by the component assigned to adatom-adatom bonds and the overlayer is found to be semimetallic. The LT spectra do not have the easily resolvable features seen in the RT spectra which may indicate that the growth is much more disordered. However, the overlayers at LT are more laminar. In addition, we present the band bending versus coverage behavior for the $\mathrm{Bi} / p$ $\operatorname{InP}(110)$ interface, which is compared to that of $\mathrm{Sb} / p-\operatorname{InP}(110)$.

\section{INTRODUCTION}

The adsorption of bismuth on $\mathrm{GaAs}^{1-4}$ has attracted interest recently. This interest stemmed from many previous studies $^{5-12}$ of the formation of interfaces between antimony and III-V semiconductors. Antimony was thought to be unique among the metals in that the first overlayer grows in an ordered way on GaAs(110). However, photoemission data and low-energy electron diffraction (LEED) results ${ }^{1,3}$ show that the same is the case for $1 \mathrm{ML}$ of bismuth on GaAs (110). The $\mathrm{Bi} / \mathrm{III}-\mathrm{V}$ interfaces thus are likely to be another group of model systems which may allow the geometric structure to be correlated with the electronic properties of the interface.

In this paper we present synchrotron radiation excited soft $\mathrm{x}$-ray photoemission spectroscopy (SXPS) results from $\mathrm{Sb}$ and $\mathrm{Bi}$ overlayers on $\operatorname{InP}(110)$. The overlayer spectra ( $\mathrm{Sb} 4 d$ and $\mathrm{Bi} 5 d$ ) carry information about the chemistry of the interface. However, it is well known that the
$\mathrm{Sb} / \mathrm{InP}(110)$ interface is unreactive $\mathrm{e}^{10,11}$ and we have recently shown that the same is true for $\mathrm{Bi} / \operatorname{InP}(110) .{ }^{13}$ Fortunately, the development of the overlayer spectra also carries information about the growth of the $\mathrm{Sb}$ or $\mathrm{Bi}$ on the semiconductor surface which has fundamental implications for the electronic structure of the interface and the height of the Schottky barrier in particular. As a method of varying the growth mode we have studied the formation of both of these interfaces as a function of temperature. We show for both interfaces that at room temperature (RT), a first layer is quickly formed and that subsequent deposition leads to strong clustering of the $\mathrm{Sb}$ or $\mathrm{Bi}$. The growth mode is therefore close to being Stransky-Krastanov. In contrast, at [low temperature (LT), $120 \mathrm{~K}$ ] our results indicate that the growth mode, again for both $\mathrm{Sb}$ and $\mathrm{Bi}$ on $\operatorname{InP}(110)$, is more laminar but very disordered. We have also measured the band bending formation of the $\mathrm{Bi} / p$-InP $(110)$ as a function of temperature and have noticed surface photovoltage effects (SPV). When corrected for SPV the band bending is 
dramatically dependent on the formation temperature and, therefore, on the growth mode of the interface. We also discuss the differences between the two interfaces and consider the implications for the Schottky barriers (SB) formed.

\section{EXPERIMENTAL DETAILS}

The SXPS experiments were performed at the TGM 2 beamline of the electron storage ring in Berlin (BESSY). The overlayer ( $\mathrm{Sb} 4 d$ and $\mathrm{Bi} 5 d$ ) core level emission spectra were taken at normal emission using a Vacuum Generators ADES 400 angle resolving spectrometer. The analyzer had an angular acceptance of approximately $4^{\circ}$. The combined resolution (light and electrons) was $0.2 \mathrm{eV}$ at the photon energies used, 55 and $45 \mathrm{eV}$. The resolution was measured from the Fermi edge of a clean gold foil in electrical contact with the sample which was also used as a reference for the Fermi level.

Clean InP(110) surfaces were prepared by cleaving aligned prenotched crystals supplied by MCP Ltd. [p-type (Zn) $1.6 \times 10^{18} \mathrm{~cm}^{-3} \mathrm{~J}$ at pressures better than $1 \times 10^{-10}$ mbar. Antimony was evaporated from a Knudsen-cell-like oven, while a commercial Knudsen cell (W.A. Technology Ltd.) was used for the bismuth evaporation. The pressure during evaporation was kept below $2 \times 10^{-10}$ mbar. The sources were located at a distance of $30 \mathrm{~cm}$ from the sample surface to minimize radiative heating effects. The evaporation rates were measured using quartz crystal balances mounted close to the sources and these were calibrated for a large thickness against a similar balance mounted at the sample position. A nominal thickness scale in monolayers (ML) which assumes layer-by-layer growth and unity sticking coefficient both on samples and balances was established ( $\mathrm{Sb}: 1 \mathrm{ML}=2.52 \AA ; \mathrm{Bi}: 1 \mathrm{ML}=2.91 \AA$ ). For both sources, stable evaporation rates between $0.02-0.2 \mathrm{ML} \mathrm{min}^{-1}$ were achieved.

\section{RESULTS AND DISCUSSION}

In Figs. 1-4 we present the overlayer core levels ( $\mathrm{Sb} 4 d$ and $\mathrm{Bi} 5 d)$ for $\mathrm{Sb} / \mathrm{InP}(110)$ and $\mathrm{Bi} / \operatorname{InP}(110)$. After normalization to the photon flux, all spectra have been analyzed with a core level fitting program which can deconvolute the spectra into as many as four spin-orbit split doublets. However, all the spectra presented here could be fitted reasonably with two or (for the highest coverages) one doublet(s). Each doublet is allowed to have an independent value for such parameters as the Doniach-Sunjic (DS) asymmetry and the Gaussian broadening.

\section{A. Sb room temperature deposition}

The $\mathrm{Sb} 4 d$ spectra taken at $\mathrm{RT}$ in Fig. 1 show clearly the symmetric growth of two features in the submonolayer spectra. At coverages $<0.5 \mathrm{ML}$ the two components of the fit have a relatively large Gaussian broadening $(\sim 0.5 \mathrm{eV})$. This reflects the inability to resolve the two features which are clearly seen at larger coverages. This broadening is caused by a range of binding energies which is in turn indicative of a range of adsorption sites for the $\mathrm{Sb}$ adatoms.

At coverages $>0.5 \mathrm{ML}$ the situation is quite different. The two clearly defined peaks shifted by $480 \mathrm{meV}$ indicate two different adsorption sites for the $\mathrm{Sb}$. We suggest that the high binding energy ( $\mathrm{BE}$ ) one is due to $\mathrm{Sb}$ bound to the substrate and the lower BE one due to $\mathrm{Sb}$ bound only to other $\mathrm{Sb}$ adatoms. The reasons for this argument were already discussed elsewhere. ${ }^{10,12}$ There it was pointed out that Sb-In bonds are more likely to be formed than $\mathrm{Sb}-\mathrm{P}$ ones because the latter are not found in nature. The assumption of preferential bonding of the $\mathrm{Sb}$ adatoms to In substrate atoms may also help to explain the unexpected Schottky barrier heights observed for $\mathrm{Sb}$ on $\operatorname{In} \mathrm{P}^{14}$ at large coverages, i.e., $<0.3$ and $>1.1 \mathrm{eV}$ for $n$ - and $p$-type InP, respectively. It should also be mentioned that the preferential bonding to the In atoms does not necessarily contradict a zig-zag chain arrangement of the $\mathrm{Sb}$ atoms in the first monolayer. An alternative zig-zag chain model which only consists of $\mathrm{Sb}-\mathrm{Ga}$ and $\mathrm{Sb}-\mathrm{Sb}$ bonds was first proposed for $1 \mathrm{ML}$ of Sb on GaAs (110) by Skeath et al. ${ }^{6}$ Since it was not favored by the experimental evidence and calculations, it was ruled out. However, more recent self-consistent calculations have shown that such a model is, in fact, possible for many $\mathrm{Sb} / \mathrm{III}-\mathrm{V}$ systems ${ }^{15}$ and that such a

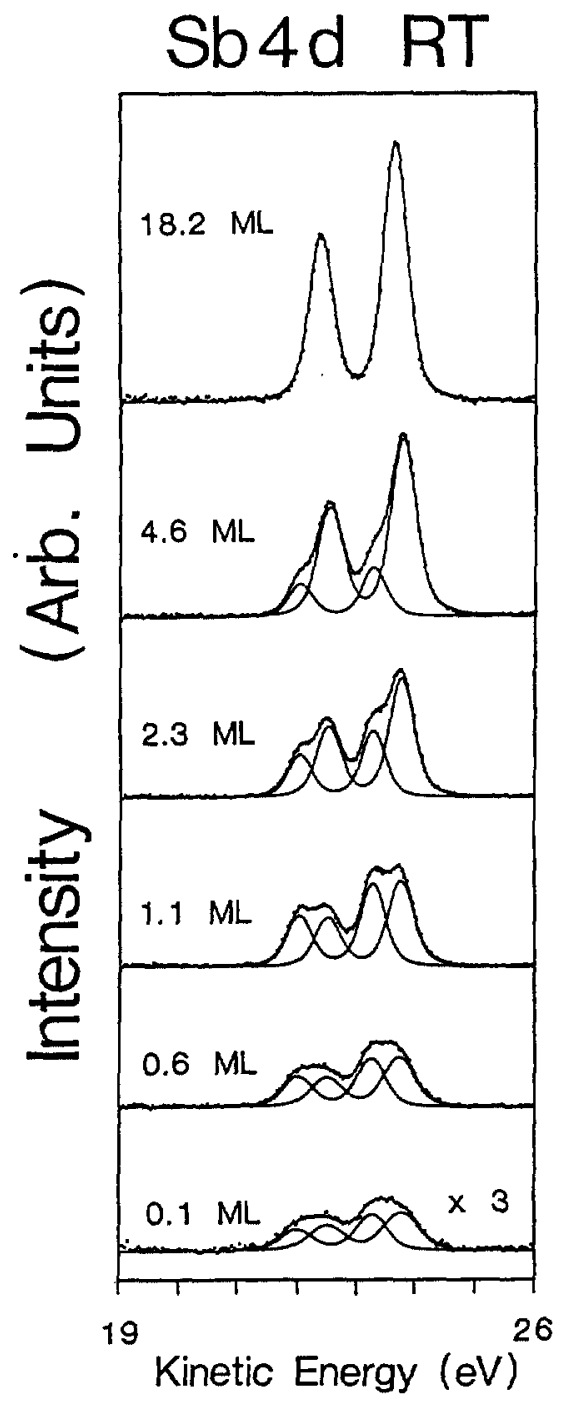

FIG. 1. Sb $4 d$ core level emission spectra for various Sb coverages deposited onto $\operatorname{InP}(110)$ at RT. Photon energy $=55 \mathrm{eV}$. 


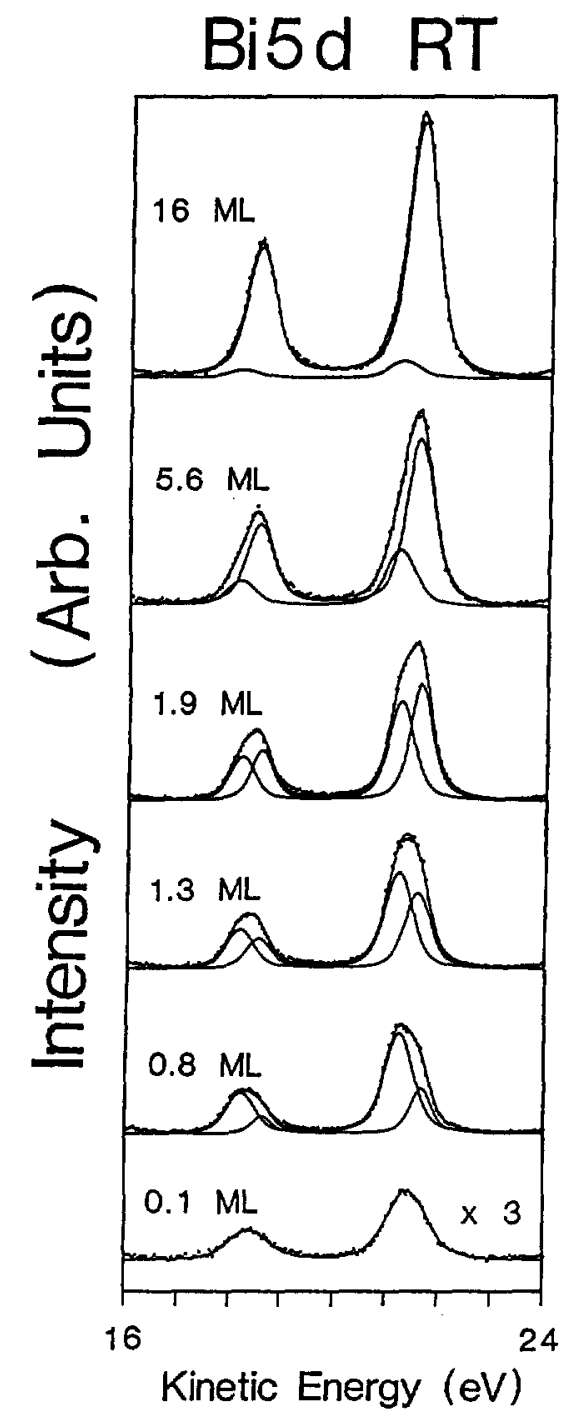

Frg. 2. Bi $5 d$ core level emission spectra for various $\mathrm{Bi}$ coverages deposited onto InP(110) at RT. Photon energy $=45 \mathrm{eV}$.

model is also consistent with the STM results. In the case of $\mathrm{Sb}$ on InP (110), a reevaluation of this model involving detailed calculations would also be useful. So far, the experimental data are not yet conclusive. For instance, the bonding of $\mathbf{S b}$ to $\mathbf{P}$ and thus the same zig-zag arrangement as for $\mathbf{S b}$ on GaAs cannot entirely be ruled out. The evolution of the $\mathrm{Sb} 4 d$ emission does appear very similar for $\mathrm{Sb}$ adsorbed on $\operatorname{GaAs}(110)^{7}$ and $\operatorname{InP}(110)$. However, in other aspects, the ordered first ML of Sb is quite different on both substrates as shown, for example, in another paper at this conference by Esser et al. ${ }^{16}$ dealing with the thermal stability of Sb overlayers on GaAs (110) and InP(110).

On deposition of more than $1 \mathrm{ML}$, the $\mathrm{Sb}-\mathrm{Sb}$ peak grows and the $\mathrm{Sb}$-substrate peak decreases in intensity as the first layer is covered. This is also shown in Fig. 5 where the relative intensity of the high $\mathrm{BE}$ peak ( $\mathrm{Sb}$-substrate) is plotted as a function of $\mathrm{Sb}$ coverage. After the increase up to about 1 ML, the intensity of this Sb-substrate peak decreases exponentially. From the slope of the attenuation, a value for the apparent electron escape depth can be derived. The large value of 7.5 ML indicates strong clustering beyond $1 \mathrm{ML}$

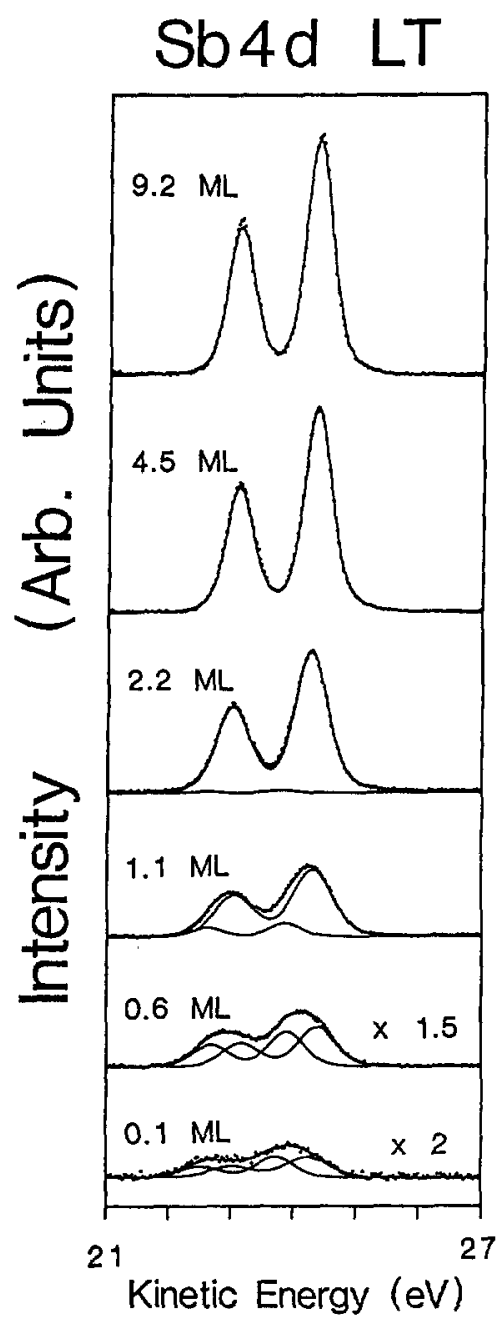

FIG. 3. Sb $4 d$ core level emission spectra for various $\mathrm{Sb}$ coverages deposited onto InP(110) at LT. Photon energy $=55 \mathrm{eV}$.

coverage. In the spectra above $6 \mathrm{ML}$, the Sb-Sb component does not require a significant DS asymmetry $(a<0.0004)$ indicating that the overlayer does not yet become metallic at these coverages. This is consistent with the Raman data ${ }^{17}$ revealing that the $\mathrm{Sb}$ overlayer at these coverages is amorphous and thus probably semiconducting. A transition to the crystalline, semi-metallic phase occurs between 10 and $20 \mathrm{ML}$.

\section{B. Bi room temperature deposition}

At very low coverages $(<0.1 \mathrm{ML})$ the development of the $\mathrm{Bi} 5 d$ core levels for $\mathrm{Bi} / \mathrm{InP}(110)$ in Fig. 2 is very similar to that of the $\mathrm{Sb} 4 d$ described above; the spectra are broad and symmetric indicating that initially the $\mathrm{Bi}$ adatoms are in a range of sites. The high coverage spectra ( $>5 \mathrm{ML}$ ) are also dominated by a component due to $\mathrm{Bi}$ bound only to other $\mathrm{Bi}$ adatoms. At coverages exceeding $20 \mathrm{ML}$ the presence of a DS asymmetry clearly reveals the semimetallic nature of the Bi. However, the spectra for coverages between 0.5 and 5 ML are significantly different from those for $\mathrm{Sb} / \mathrm{InP}(110)$. This can already be judged from the raw data.

Below $1 \mathrm{ML}$, the spectra are asymmetric with the spectral 

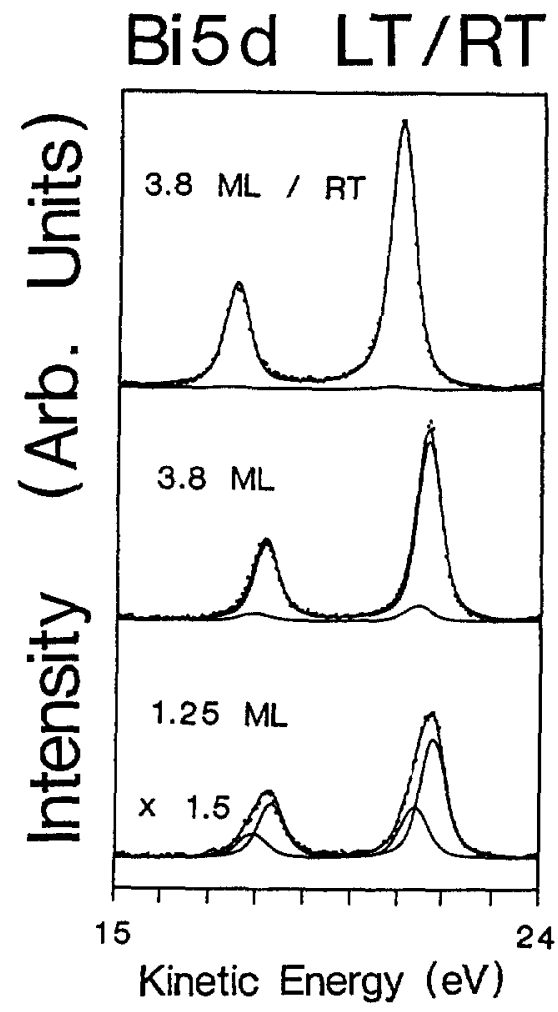

FIG. 4. Selected Bi $5 d$ core level spectra for the LT deposition onto InP (110) and the warming up to RT. Photon energy $=45 \mathrm{eV}$.

weight to the high $\mathrm{BE}$ side. As more bismuth is deposited, the spectral weight shifts to the low BE side with the spectra being almost symmetric but broad around $1.5 \mathrm{ML}$. This situation is in a clear contrast to $\mathrm{Bi} / \mathrm{GaAs}(110)^{1,3}$ and $\mathrm{Sb} / \mathrm{InP}(110)$ described above. The difference is even more evident in the evolution of the pair of spin-orbit split doublets fitted to the data. In the cases of $\mathrm{Bi} / \mathrm{GaAs},{ }^{1} \mathrm{Sb} / \mathrm{GaAs},{ }^{7}$ and $\mathrm{Sb} / \mathrm{InP}$, both the low and high $\mathrm{BE}$ components are found to grow together until $1 \mathrm{ML}$. In the $\mathrm{Bi} / \mathrm{InP}$ spectra presented the high $\mathrm{BE}$ component, again assigned to $\mathrm{Bi}-$ substrate bonds, is stronger than the low $\mathrm{BE}$ one (shifted by $0.4 \mathrm{eV}$ ) up to approximately $1.5 \mathrm{ML}$. The high $\mathrm{BE}$ one reaches its maximum intensity around $0.8 \mathrm{ML}$. At that coverage the amount displayed by the high $\mathrm{BE}$ component is equivalent to approximately $0.5 \mathrm{ML}$, i.e., the amount needed to cover one of the substrate species. Therefore, it seems reasonable to assume that $\mathrm{Bi}$ is preferentially bonded to only one of the substrate species. Unlike the $\mathrm{Sb} / \mathrm{InP}$ system for which one can only speculate about the possibility of preferential bonding, the evolution of the $\mathrm{Bi} 5 d$ emission is a good indication for the preferential bonding. On simple chemical arguments that Bi does not form bonds to $P$ the In atom is a more likely choice for a single adsorption site, as we have suggested for $\mathrm{Sb} / \mathrm{InP}{ }^{10,12}$

The relative intensity of the $\mathrm{Bi}$-substrate peak is also plotted in Fig. 5 as a function of Bi coverage. After reaching its maximum, the decrease of the intensity may be divided into two steps. First, a fast decrease until about $2.5 \mathrm{ML}$ followed by a decrease with a lower slope than that of $\mathrm{Sb}$. The former is indicative of a more laminar growth up to $2.5 \mathrm{ML}$. It was
Rel. Intensity of the High BE Peak

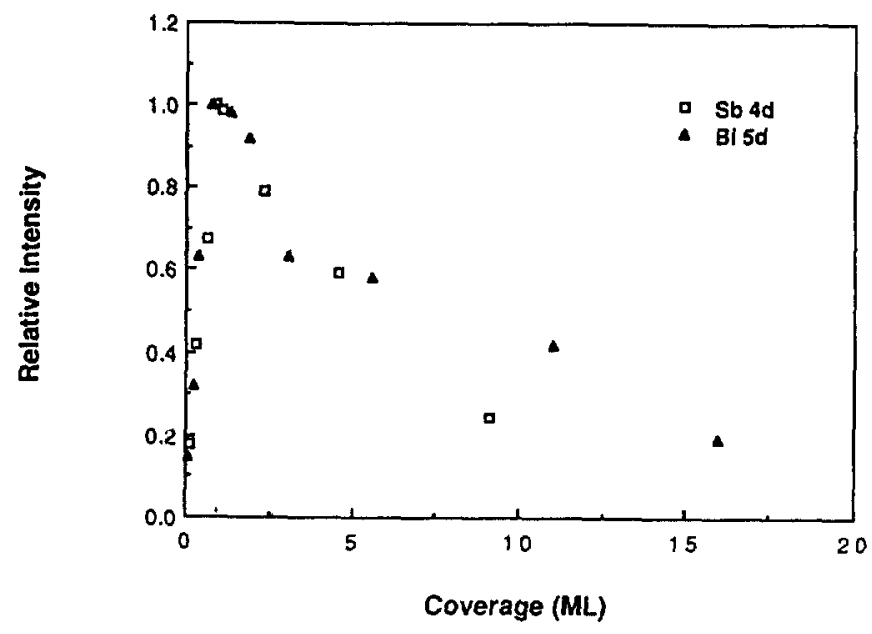

FIG. 5. Relative intensity of the high BE component which is obtained from the curve fitting procedure and assigned to the adatom-substrate bond as a function of coverage. Maximum intensity $=1$. Sb: open squares, Bi: full triangles.

also argued for $\mathrm{Bi}$ on GaAs that $\mathrm{Bi}$ grows in a layer-by-layer fashion up to about $2.5 \mathrm{ML}$ before the onset of island growth.' Thereafter, the degree of clustering is larger than is observed for $\mathrm{Sb} / \mathrm{InP}(110)$. Here, the apparent escape depth is $11 \mathrm{ML}$. The same behavior was found in the In $4 d$ intensity attenuation curves for $\mathrm{Sb}$ and $\mathrm{Bi}$ coverage. The stronger ability of clustering for Bi simply reflects a higher mobility on the InP surface at RT.

\section{Sb and Bi low temperature deposition}

The $\mathrm{LT}$ results for $\mathrm{Sb}$ are presented in Fig. 3. The core levels at low coverages are broader than those at RT, requiring the relatively high value of the Gaussian broadening $(0.5$ $\mathrm{eV}$ ) in the fits. Again, this broadening is explained by a range of binding sites for the $\mathrm{Sb}$ adatoms leading to a range of binding energies. At higher coverages the core levels are different from those at RT in that the broadening remains large throughout the coverage range. At coverages $>1 \mathrm{ML}$ the low $\mathrm{BE}$ component increases and at higher coverages dominates the spectra. This component is again attributed to $\mathrm{Sb}$ bound only to other $\mathrm{Sb}$ atoms.

The Bi core levels at low coverages show a similar evolution to the $\mathrm{Sb} 4 d$ levels in Fig. 3 and can be fitted in the same way with two broad peaks representing a range of binding energies. At the somewhat higher coverages, the high $\mathrm{BE}$ peak also loses intensity relative to its low $\mathrm{BE}$ partner as the $\mathrm{Bi}-\mathrm{Bi}$ component grows. When the $\mathrm{Bi}$ overlayer of $3.8 \mathrm{ML}$ was warmed to RT the fitted high BE component was found to decrease to a negligible intensity.

It must be clearly stated that the LT core levels (both $\mathrm{Sb}$ and $\mathrm{Bi}$ ) are not suitable candidates for the fitting procedure as they do not show distinct features in the same way as the RT ones do. We have also achieved reasonable fits of the low coverage spectra with only one very broad component $(0.7$ $\mathrm{eV}$ ) to represent the range of binding energies. With this analysis the asymmetry in the spectra $>1 \mathrm{ML}$ must then be 
taken care of by the introduction of a new component to higher $\mathrm{BE}$. A third possibility would be one with only one doublet throughout the coverage range but with a relatively large DS factor at the higher coverages. This proved not to yield reasonable fits in either the $\mathrm{Sb}$ or the Bi case.

In both cases the growth of the overlayer at $\mathrm{LT}$ is found to be very laminar as judged by the In $4 d$ intensity attenuation. ${ }^{10,13}$ At LT electron escape depths of 1.5 and $1 \mathrm{ML}$ are evaluated for $\mathrm{Sb}$ and $\mathrm{Bi}$, respectively. These values are in good agreement with the empirical data by Seah and Dench. ${ }^{18}$

\section{Band bending at the $B i / \ln P(110)$ interface}

In a previous study ${ }^{13}$ it was shown that there are no chemical shifts in the In $4 d$ spectra when $\mathrm{Bi}$ is deposited and that the interface thus is abrupt. The rigid shifts in the In $4 d$ core level spectra can be attributed to the band bending at the interface. Furthermore, the strong clustering of Bi at RT allows the substrate core level emission and thus the band bending to be monitored up to nominal deposition thicknesses of several tens of ML. The position of the interface Fermi level in the band gap is shown in Fig.6. It is apparent from the RT data that the band bending does not reach its final value at $1 \mathrm{ML}$ coverage since a decrease of about 100 $\mathrm{meV}$ is found for coverages $>1 \mathrm{ML}$. The final position of the Fermi level [0.8 eV above valence-band maximum (VBM)] is only approached for coverages beyond the onset of metallicity ( $>20 \mathrm{ML}$ ). This behavior is in good agreement with the band bending derived from electric field induced Raman scattering (EFIRS). ${ }^{19}$ However, the position of the maximum band bending in the low coverage range is slightly shifted and the minimum beyond is more pronounced in the EFIRS data. A possible reason for the latter could be the fact that the photoemission data represent an average over almost the entire sample area, whereas the Raman information is only collected within an area of less than $100 \mu \mathrm{m}$ in

\section{$B 1 / p-\operatorname{In} P(110)$}

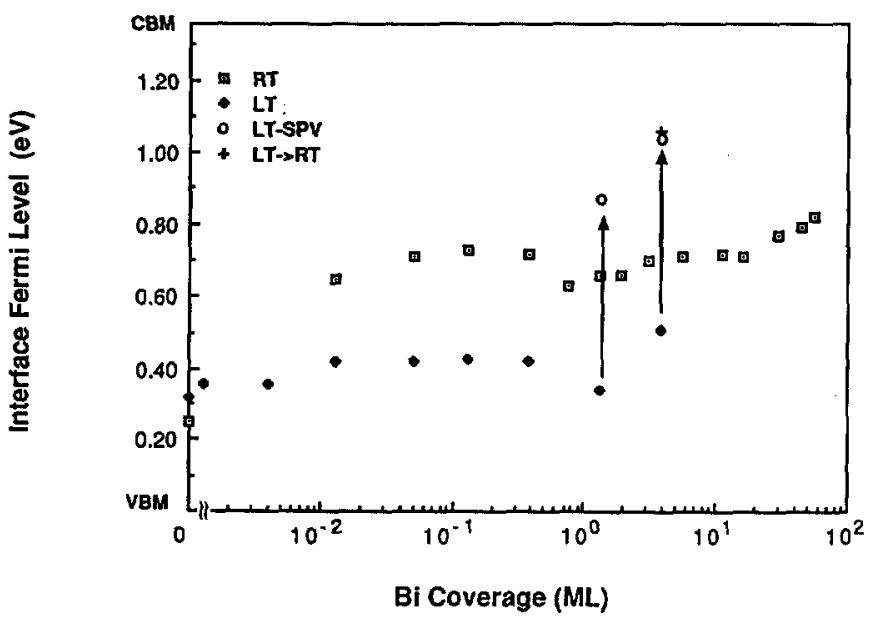

FIG. 6. Position of the interface Fermi level in the band gap as a function of coverage for RT and LT deposition of Bi onto $p$-type InP. Also indicated are the SPV corrections of the LT data and the position at RT after a LT deposition. diameter. The variation of band bending below $20 \mathrm{ML}$ is likely to be induced by imperfections in the overlayer $(<1$ ML) and partial healing of those ( $>1 \mathrm{ML}$ ). The Schottky barrier of $0.8 \mathrm{eV}$ at the highest coverages is in good agreement with the EFIRS data as well as electrical measurements of thick Bi contacts to InP $(110)^{19}(I-V: 0.83 \mathrm{eV}$ and $C-V: 0.85 \mathrm{eV}$ ). In addition, the Schottky barrier height for Bi on $n$-type InP was measured to be $0.48 \mathrm{eV}$ by $I-V .{ }^{19}$ When this is considered with the theoretical pinning level of 0.76 eV calculated by Tersoff, ${ }^{20}$ it seems that a metal induced gap state (MIGS) model can explain the final pinning position of the Fermi level. This is in contrast to the Sb case for which a modified MIGS model which takes the exact bonding at the interface into account may be more appropriate.

An interesting effect occurs at the LT deposition of Bi. There is emission of photoelectrons above the reference Fermi level as measured from a gold foil in electrical contact with the sample. This was also observed previously by Alonso et al. ${ }^{21}$ and they explained this phenomenon as being due to a surface photovoltage (SPV) effect. Hence the interface Fermi level position has to be corrected for SPV. As can be seen in Fig. 6, the values after SPV correction give rise to Schottky barriers larger than $1 \mathrm{eV}$. These surprisingly high barriers are no longer in accordance with the MIGS model. It is also worth mentioning that the same barrier is found after warming up to RT, where the SPV effect is negligible. The reason for this behavior is not clear, but one may again speculate that the detailed nature of the bonding being somewhat different for LT deposition has a large effect on the formation of the Schottky barrier. This is an important result as it implies that the method of interface preparation plays a crucial role in determining the final SB height. The possibility arises that by choosing the formation temperature carefully, the SB at the interface when warmed to RT may be tuned to a desired height. This possibility requires further study.

\section{SUMMARY}

The evolution of the $\mathrm{Sb} 4 d$ and $\mathrm{Bi} 5 d$ core level emission intensities reveals a pronounced difference for the adsorption of $\mathrm{Sb}$ and $\mathrm{Bi}$ onto clean cleaved $\operatorname{InP}(110)$ at RT. Two spin-orbit splitted doublets are sufficient to curve fit the experimental data. The high $\mathrm{BE}$ component may be assigned to the adatom-substrate bond and the low BE one to the adatom-adatom bond. While this assignment is not conclusive for the $\mathrm{Sb}$ case, the Bi $5 d$ spectra can be easily interpreted in this sense. Then the $\mathrm{Bi}$ is likely to be bonded preferentially to one substrate species which is presumably In. For $\mathrm{Sb}$, the evolution of the $\mathrm{Sb} 4 d$ emission is very similar to that of $\mathrm{Sb}$ on GaAs where the two components obtained from curve fitting were interpreted in terms of $\mathrm{Sb}-\mathrm{Ga}$ and $\mathrm{Sb}-\mathrm{As}$ bonds. However, there seems to be enough indirect evidence to question whether the same is the case for $\mathrm{Sb}$ on InP. There is a need for a theoretical reevaluation of alternative models for the bonding of the group V elements to InP(110), such as that proposed by Skeath et al. ${ }^{6}$

The RT evolution of the band bending at the $\mathrm{Bi} / \mathrm{p}-\mathrm{InP}$ interface demonstrates that the final pinning position is not 
reached at $1 \mathrm{ML}$ coverage but that as much as $60 \mathrm{ML}$ are needed before the Schottky barrier formation is completed. The RT data are consistent with a MIGS model.

The LT data are characteristic of a more disordered but also more laminar adsorption. In terms of band bending, the LT experiments have revealed that SPV effects occur at the $\mathrm{Bi} /$ InP interface and have to be taken into account for the evaluation of the correct band bending. The final coverage SB height even after warming up to RT is found to be different from that formed at RT. The barrier height cannot be explained by a simple MIGS model. The exact nature of the bonding at the interface is likely to have this pronounced effect on the barrier height.

\section{ACKNOWLEDGMENTS}

This work was funded by the European Community under its Stimulation Programme (Contract No. SJ-2J-0210). Additional support from the BMFT (FRG) (Contract No. $05230 \mathrm{NUI}$ ) is also acknowledged. The authors would like to thank N. Esser and U. Resch for useful discussions and Dr. K. Horn, Fritz-Haber Institut, Berlin for the use of his core level fitting program.

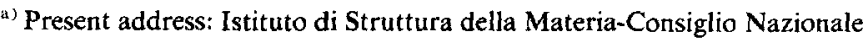
delle Ricerce, Via E. Fermi, I-00044 Frascati, Italy.

'J. J. Joyce, J. Anderson, M. M. Nelson, C. Yu, and G. J. Lapeyre, J. Vac. Sci. Technol. A 7, 850 (1989).

${ }^{2}$ S. Horng and A. Kahn, J. Vac. Sci. Technol. B 7, 931 (1989).
}

${ }^{3}$ A. Taleb-Ibrahimi, R. Ludeke, R. M. Feenstra, and A. B. McLean, J. Vac. Sci. Technol. B 7, 936 (1989).

${ }^{4}$ N. Esser, M. Hünermann, U. Resch, D. Spaltmann, J. Geurts, D. R. T. Zahn, W. Richter, and R. H. Williams, Appl. Surf. Sci. 41/42, 169 (1989). ${ }^{5}$ C. B. Duke, A. Paton, W. K. Ford, A. Kahn, and J. Carelli, Phys. Rev. B 26, 802 (1982).

${ }^{6}$ P. Skeath, C. Y. Su, W. A. Harrison, I. Lindau, and W. E. Spicer, Phys. Rev. B 27, 6246 (1983).

'F. Schäffler, R. Ludeke, A. Taleb-Ibrahimi, G. Hughes, and D. Rieger, J. Vac. Sci. Technol. B 5, 1048 (1987).

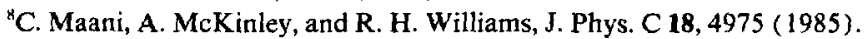

"C. Mailhiot, C. B. Duke, and D. J. Chadi, Phys. Rev. B 31, 2213 (1985).

${ }^{16}$ D. R. T. Zahn, W. Richter, A. B. McLean, R. H. Williams, C. Stephens, I. T. McGovern, R. Cimino, W. Braun, and N. Esser, Appl. Surf. Sci. 41/42, 179 (1989).

${ }^{1}$ T. Kendelewicz, R. Cao, K. Miyano, I. Lindau, and W. E. Spicer, J. Vac. Sci. Technol. B 7, 991 (1989).

${ }^{12}$ R. H. Williams, D. R. T. Zahn, N. Esser, and W. Richter, J. Vac. Sci. Technol. B 7, 997 (1989).

${ }^{13}$ C. Stephens, D. R. T. Zahn, R. Cimino, W. Braun, K. Fives, and I. T. McGovern, Vacuum (in press).

${ }^{14}$ D. R. T. Zahn, A. B. McLean, R. H. Williams, N. Esser, and W. Richter, Appl. Phys. Lett. 52, 739 (1988).

'5J. P. LaFemina, C. B. Duke, and C. Mailhiot, J. Vac. Sci. Technol. B 8, 888 (1990).

${ }^{16}$ N. Esser, M. Reckzügel, R. Srama, U. Resch, D. R. T. Zahn, W. Richter, C. Stephens, and M. Hünermann, J. Vac. Sci. Technol. B 8, 680 (1990).

"N. Esser, U. Resch, U. Rettweiler, W. Richter, D. R. T. Zahn, and R. H. Williams, Vacuum 38, 229 (1988).

${ }^{18}$ M. P. Seah and W. A. Dench, Surface Interface Anal. 1, 2 (1979).

${ }^{14}$ D. R. T. Zahn, N. Esser, W. Richter, C. Stephens, and R. H. Williams, J. Phys., Condens. Matter 1, SB45 (1989).

${ }^{20}$ J. Tersoff, Phys. Rev. B 32, 6968 (1985).

${ }^{21}$ M. Alonso, R. Cimino, and K. Horn, Phys. Rev. Lett. 64, 1947 (1990). 